\title{
Wild type
}

D H K K T I S S A I I N H A F L Q N T V M K N C N Y K R K R R E R D W D C N T K K D V C I P D R R Y Q L C M K E L T N L V N N T D T N F H R D I T F R K L Y L K R K L I Y D A A V E G D L L L K L N N Y R Y N K D F C K $D$ D I R W S L G G D I I M G T D M E G I G Y S KVV E N N L R S I F G T D E K A Q Q R R K $Q$ W W N E S K A Q I W T A M M Y S V K K R L K G N F I W I C K L L N V A V N I E P $Q$ I $Y$ R W I R E W G R D Y V S E L P T E V Q K L L K E K C C D G K I N

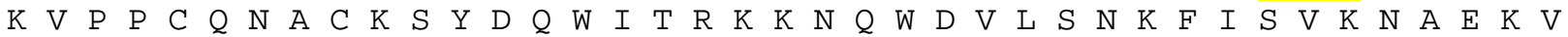
Q T A G I V T P Y D I L K Q E L D E F N E V A F E N E I N K R D G A Y I E L C V C S V E E A K K N T Q E V V T N V D N A A K S Q A T N S N P I S Q P V D S S $K A E K \vee P$

\section{STBP glycan}

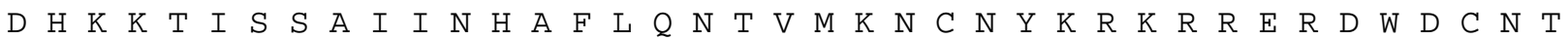

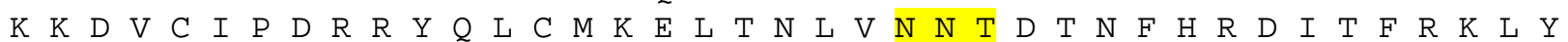

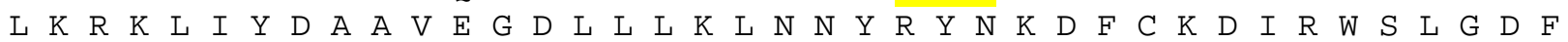

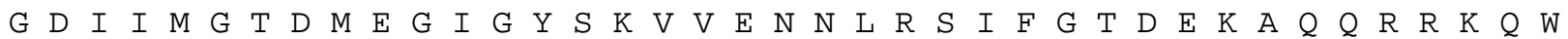
W N E S K A Q I W T A M M Y S V K K R L K G N F N W T C C K L N

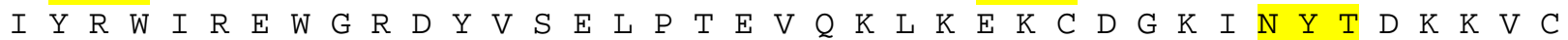

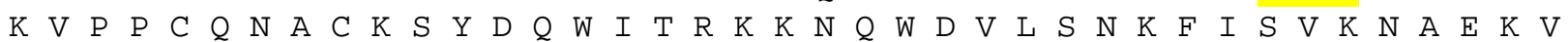

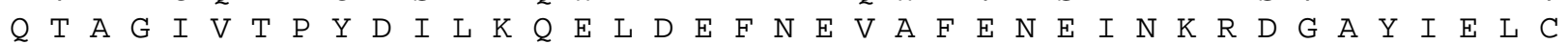
$V$ C S V E E A K K N T Q E V V T N V D N A A K S Q A T N S N P I S Q P V D S S $K A E K V P$

P1

D H K K T I S S A I I N H A F L Q N T V M K N C N Y K R R K R R E R

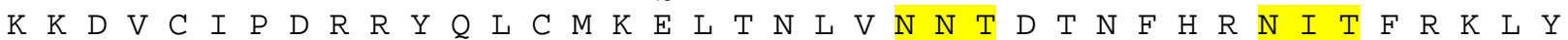

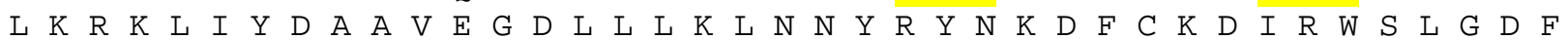

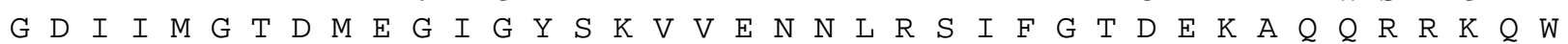
W N E S K A Q I W T A M M Y S V K K R L K G N F I W I C C K L

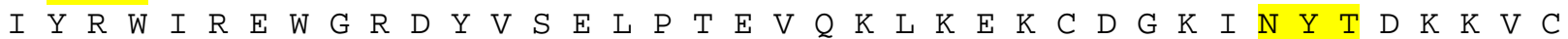
$\begin{array}{lllllllllllllllllllllllllllllllllllllll}K & V & P & P & C & Q & N & A & C & K & S & Y & D & Q & W & I & T & R & K & K & N & Q & W & D & V & L & S & N & K & F & I & S & V & K & N & A & S & K & V\end{array}$

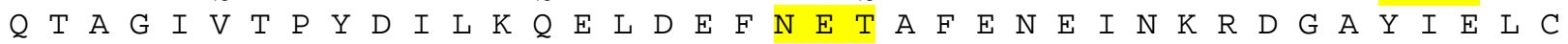
V C S V E E A K K N T Q E V V T N V D N A A K S Q A T N S N P I S Q P V D S S $K A E K \vee P$

\section{Max}

D H K K T I S S A I I N H A F L Q N T V M K N C N Y K R K R R E R D W D C N N

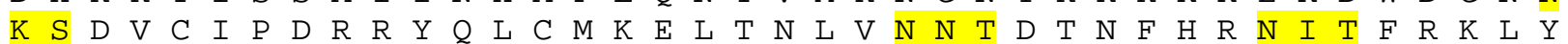

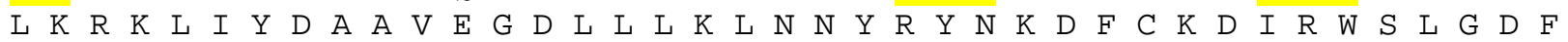

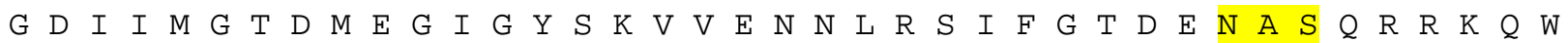
W N E S K A Q I W T A M M Y S V K K R L K G N $F$

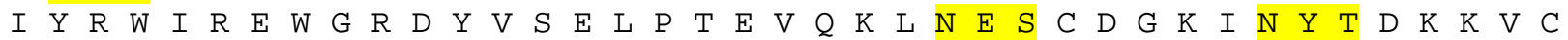

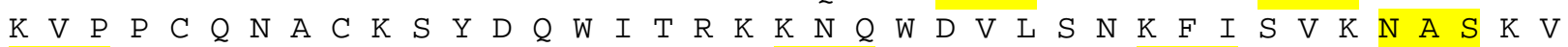
N T T G I V T P V C S V E E A K K N T Q E V V T N V D N A A K S Q A T N S N P I S Q $K A E K V P$ 